\title{
A qualitative study on the pros and cons of ethnically matching culturally and linguistically diverse (CALD) client families and child protection caseworkers
}

\begin{abstract}
A review of the literature indicated that empirical research into the advantages and disadvantages of ethnically matching child protection caseworkers and culturally and linguistically diverse (CALD) client families is not extensive. The main aim of this study was to address this gap. Qualitative interviews were conducted with 29 CALD client families and 17 child protection caseworkers (as part of a larger study). It was found that if CALD families fear a breach of confidentiality (because of cultural reasons to protect the family's privacy and name), and this fear exceeds the two main benefits of ethnic matching - namely, that it helps overcome language barriers and provides (cultural) sensitivity - then they are likely to prefer a non-matched caseworker. This may be a challenge in child protection practice if the pool of culturally competent caseworkers is small. Another challenge identified was when CALD families preferred an ethnic match but was seen by case managers as a risk to the accurate assessment of a child's wellbeing because the caseworker was over-identifying with the client family. Thus, the provision of a culturally sensitive service can be at odds with the need to protect children from harm. This issue is seen as best addressed on a case by case basis. This study is significant because it has shown that 'over-identification' is an important work practice issue in this matter. It has also used empirical evidence to inform best practice principles regarding ethnic matching.
\end{abstract}

Keywords: Ethnic matching, child protection, cultural competency, over-identification, culturally and linguistically diverse (CALD), caseworkers 


\subsection{Background}

In countries like Australia, New Zealand, UK, USA, and Canada, where the white English speaking forms the mainstream, families of culturally and linguistically diverse (CALD) backgrounds differ from the majority in culture and/or language. To help overcome some of the barriers that may result from these differences, both CALD families and caseworkers in the child protection system (CPS) may prefer (to provide) a caseworker from the same ethnic background as the client family (e.g. Harris \& Hackett, 2008; Chand, 2005; Weinfeld, 1999). Perry and Limb (2004) report that in California, caseworkers of various ethnic backgrounds (including American-Indian, Hispanic/Latino(a), Caucasian, Asian-American, and African-American), were between 1.28 and greater than 2 times more likely to have a race matched client. Thus, the results of this study suggest that ethnic matching could be a relatively common practice.

Yet despite this, a review of the literature indicated that there is little empirical research into the advantages and disadvantages of ethnically matching child protection caseworkers and client families. Indeed, the study by Perry and Limb (2004) is the only known quantitative study conducted on the prevalence of ethnic matching. Thus, only a few known empirical studies have been conducted in this area (Gray, 2002; Harris \& Hackett, 2008; Ryan, Garnier, Zyphur, \& Zhai , 2006; Chuan \& Flynn, 2006; Perry \& Limb, 2004; Maitra, 2003; Weinfeld, 1999), even though the theoretical literature commonly cites race matching as an effective strategy for meeting the cultural and linguistic needs of CALD families ${ }^{1}$ (e.g. Osterling, D'andrade, \& Austin, 2008; Sale, 2006; Chand, 2005; Chand \& Thoburn, 2005; Korbin, 2002; Chand, 2000; Owusu-Bempah, 1999). Thus, little is known about whether ethnic matching does in fact overcome barriers that arise from cultural and linguistic differences and/or whether it introduces barriers to effective child protection practice. Moreover, little has changed in the last 15 or so years when Courtney et al (1996) argued that little is known about the effects of same-race service provision on outcomes for children of colour in the USA because virtually no such empirical evidence exists. The aim of this study was to help address this gap in the knowledge base by conducting empirical research.

\subsection{Literature review: What do we know so far about the pros and cons of ethnically matching} caseworkers and client families?

\footnotetext{
${ }^{1}$ Note: This paper has not referenced literature that reports on ethnic matching between CALD children and foster carers and adoptive parents, which in comparison is more extensive than the literature on ethnic matching between caseworkers and client families.
} 
Caseworkers may be matched with CALD clients because their shared ethnicity, including a common language, culture and/or experience as an ethnic minority, can help facilitate engagement with the child protection process (e.g. Osterling et al. 2008; Gray, 2002). Similarly, Weinfeld (1999), who conducted qualitative interviews with 45 health care and social service professionals in Montreal of non-British and non-French ethnic origin, (and whose study was the first known empirical investigation into ethnic matching), concluded from his study that the three main advantages of ethnic matching were language competence, cultural competence and trust; all of these can facilitate engagement with the client family.

Ethnic matching can also enhance accuracy in the assessment and understanding of a child's well-being (e.g. Gray, 2002; Weinfeld, 1999). For example, Owen and Farmer (1996, cited in Chand, 2005, p. 811) report that:

In some Asian languages (in the UK) the words necessary for the description of sexual abuse do not exist, or are so rarely used that people would be shocked by their usage ... [And so] in many cases a balance had to be maintained between politeness and clarity.

Thus, ethnically-matched bilingual staff can play an important role in understanding cultural nuances in interpretations and explanations about abuse or neglect, and in turn are able to more accurately represent the voices of CALD families who may otherwise feel or be misunderstood by a non-ethnically matched caseworker.

The increased engagement and accuracy that ethnic matching offers is implicitly built on the assumption that "a child welfare worker who is the same as the child's ethnic background will demonstrate less bias towards the client" (Harris \& Hackett, 2008, p. 203). However, the research does not necessarily support this. For example, in an extensive literature review, Courtney et al (1996) found no evidence that race-matching was effective in improving outcomes for children of colour in the USA (cited in Harris \& Hackett, 2008, p. 203). Korbin (2002, p. 639) argues that caseworker bias may occur because:

Even if social workers share the same cultural group, differences in education, SES (socio-economic status), gender, age or other life experiences may cause substantial communication and interpretation barriers that must be overcome in a similar manner as if the two were from different cultures.

Moreover, caseworker bias may not just be in the direction of increased judgement of that family, but in the other direction as well; namely that caseworkers minimise risk of harm because they have increased empathy for 
that family ${ }^{2}$. Thus, over-identification of a caseworker to a matched client family means that there are additional benefits to non-matching. This point has only been made once in the empirical literature by Gray (2002) who conducted qualitative interviews with 22 client families and caseworkers in London. He reports that "managers provided supervision and informal debriefing sessions after (home) visits to ensure that families did not transfer their problems onto workers ... (matched) in terms of ethnicity, gender and parenthood, which might otherwise lead to over-identification" (p. 17).

The scant literature also indicates that a significant benefit of non-matching is that it can help overcome any fear that CALD families have about a breach in confidentiality, even though it is mandatory for all staff to keep matters confidential except as required by law (e.g. Chand \& Thoburn, 2005; Maitra, 2003; Korbin, 2002). As an example, Brandon et al (1999) conducted a qualitative project on 29 children of ethnic minority heritage in London, involving the recruitment of South Asian support workers to work with families of a similar ethnic and cultural background. They found that "despite valuing their ability to speak the same language and understand their culture and religion some (families) experienced their support as intrusive and were concerned about possible lack of confidentiality" (cited in Chand \& Thoburn, 2005, p. 174). The loss of privacy is a cultural factor common in collectivist cultures where family name and standing in the community are important.

Thus, despite the increased engagement and accuracy that can ethnic matching offer, non-ethnic matching may be in the better interest of a CALD family. This is because it avoids possible caseworker bias (in the form of either increased judgment or increased empathy) and overcomes any fear that CALD families have of a breach in confidentiality.

Given that there are significant benefits for CALD families in non-matching, training in cultural competency for non-CALD caseworkers becomes crucial. Several authors make this point. For example, Maitra (2003) says, “culturally expert assessments require more than culturally matched experts" (p. 64). Owusu-Bempah (1999, pp. 29-30) points out that,

\footnotetext{
2 The process of increased familiarity and empathy leading to risk minimisation is known as 'overidentification', and is acknowledged in child protection work as an issue, especially in serious case reviews when risks are not appropriately responded to. It can occur between any two people and in any context (e.g. between parent and child, between a caseworker who had a mother with depression and a case they are investigating in which the mother has diagnosed depression, etc.). However, in the context of ethnic matching, only one reference in the literature was identified (Gray, 2002).
} 
Even those whom one would expect to be more understanding or sympathetic towards ethnic minority children and families' circumstances sometimes propagate ideas or perspectives damaging to their well-being. This raises the pertinent question as to whether the recruitment of more ethnic minority professionals by itself will improve the situation for ethnic minority clients ... [Thus] their most valuable contribution would appear to be one of helping other colleagues to develop an understanding of, and respect for, ethnic minority cultures and cultural practices.

Finally, Chand and Thoburn (2005, p. 177) point out that cultural competency training is essential for all workers because:

Even if workers are matched for race, the diversity of the UK means that all workers are likely to be providing services to families whose language, cultural heritage, social background, and/or religious affiliation differ in at least some important respects from their own.

Thus, responsibility for work with CALD families should not be placed solely on CALD caseworkers; "the responsibility for addressing ethnicity and racism should be shared by all workers, black and white" (Chand, 2000, p. 76). Indeed, from the training course he runs on race, diversity, and child protection in Bristol, Dugdale (2006) has found anecdotal evidence of mainly white social work teams viewing a black colleague as 'the expert' on ethnic minority issues instead of being informed themselves (cited in Sale, 2006, p. 29). Similarly, Barn, Sinclair \& Ferdinand (1997) report from their study of the child protection register of three local authorities in London and the Midlands (that "white managers and practitioners emphasised their lack of cultural awareness as a weakness when working with black families, whilst black managers and practitioners argued that race and ethnicity were not adequately taken into account due to Eurocentric child protection procedures" (cited in Chand, 2000, p. 69). Thus, ethnic matching may be 'a quick band-aid solution' in the need for cultural knowledge, but training in cultural competency helps address this need and avoids the use of an absolutist or universal approach to the assessment of risk across cultures. Moreover, an over-reliance on CALD caseworkers as 'cultural experts' can be a resource issue because it can unequally distribute caseloads in especially highly culturally diverse areas.

Finally, Korbin (2002, p. 639) makes the point that "power differentials inherent in a clinical encounter between provider and recipient may also pose barriers among those that share a cultural tradition". Thus, ethnic matching may not even be in the best interest of a CALD caseworker. 
In short, ethnic matching can be both effective and ineffective. It is effective for overcoming language barriers, understanding culture better, improving trust in and engagement with the child protection system, and enhancing accuracy in risk of harm assessments for children across different cultural backgrounds. However, it is ineffective if matched workers are more or as judgemental than non-matched caseworkers, if matched caseworkers over-identify with their client families, and/or if CALD families fear a breach of confidentiality. It also seems that the best way to address this complexity is through training in cultural competency, which has a number of benefits and is seen as "a must for all staff" (Sale, 2006, p. 29). Cultural competency training allows for the provision of a non-match when CALD families request this due to a fear of a breach of confidentiality, without compromising the provision of a culturally aware and sensitive service; it avoids power differentials between service users and providers that could lead to aversive consequences for CALD caseworkers; it eases resource burdens especially in CALD-dense areas; and it avoids using a standardised assessment that is insensitive to cultural differences in parenting and family functioning.

\subsection{Aims and significance of this study}

Given the complexity of this issue and that the current research base is small, qualitative research is an appropriate and useful tool for exploring and identifying key themes and relevant issues. Thus the first main strength of this study is that it uses semi-structured qualitative interviews to allow for the in-depth exploration of themes, perceptions and experiences in ethnic matching caseworkers and client families. This methodology can help identify whether themes in the (albeit scant) literature are replicable or not and whether there are any new findings (the aim of this study).

The other main advantage of this study is that it interviews both CALD families as well as caseworkers who service them, so that responses can be compared and triangulated. In doing so, consistency between services users and service providers can be identified and used to provide evidence for the continued use of that particular practice, and inconsistency can provide insight on how to overcome any gaps in current service delivery and in turn better inform best practice principles when working with CALD families.

This study was carried out as part of a larger study on the needs of CALD families in the New South Wales (NSW) CPS in Australia and how best to meet them. This larger study was partly funded by the NSW 
Department of Human Services $\left(\mathrm{DHS}^{3}\right.$ ), was conducted between 2007 and 2010, and was comprised of three stages - a literature review, case file reviews, and qualitative interviews. The qualitative interviews covered a range of topics including cultural norms on parenting and family functioning, CALD representation and service provision in the CPS, cross-cultural service provision, ethnically-matching CALD families and caseworkers, the use of interpreters, examples of cultural and non-cultural in/appropriate practice, barriers to culturally appropriate practice and suggestions for improving culturally appropriate practice. Only data that pertains to the perceived and experienced advantages and disadvantages of ethnic matching, collected during the interviews, have been presented here.

\section{Method}

\subsection{Recruiting participants}

\subsubsection{CALD families}

DHS caseworkers and case managers at five of ten Community Service Centres (CSCs) participating in this study were asked to approach their current CALD clients to see if they were interested in taking part in an interview. These five CSCs were selected because of the high CALD population density in these areas, which was seen to increase the effectiveness of the recruitment process. Also, this is an arms-length approach consistent with ethics protocol that minimises perceived coercion to participate ${ }^{4}$. If possible CALD participants were interested in taking part, a suitable time and place for the interview was then set up.

Parents and carers from any CALD background were invited to take part to help ensure that the target sample size was met $(\mathrm{n}=30)$. In total, 29 CALD parents/carers (henceforth referred to as 'CALD families') took part in the study. Thus the target sample was reached. Two participants opted to conduct the interview in a private room at the DHS office and the remainder chose to conduct the interview at their home. Interviews varied in length from 15 minutes to 1 hour and 50 minutes (Mean = 45 minutes). Five interviews were conducted with interpreters, which the local CSC assisted in organising.

\subsubsection{DHS caseworkers and case managers}

\footnotetext{
${ }^{3}$ At that time, DHS was known as the Department of Community Services (DoCS). The other funding body was the Social Policy Research Centre (SPRC) at the University of New South Wales (UNSW).

${ }^{4}$ Ethics approval from the Human Research Ethics Committee (HREC) at the UNSW was obtained in November 2007 for all stages of this three year project.
} 
DHS caseworkers and case managers at eight of the ten participating CSCs were also invited to take part in the study (two CSCs were excluded as themes began to saturate). Again using an arms-length approach, case managers in each CSC were asked to approach two caseworkers on behalf of the researcher to see if they would be interested in taking part. Case managers themselves were also invited to take part.

In total, 17 caseworkers and case managers took part in this study. Originally, there was a target sample of 20 interviews with caseworkers. However, as themes began to saturate it was no longer necessary to continue conducting the interviews. All interviews were conducted in a private room at the DHS office. Interview times ranged from 54 minutes to 2 hours and 10 minutes (Mean $=1$ hour and 11 minutes).

\subsection{Conducting and theming interviews}

At the beginning of each interview, an Information Statement and Consent Form was provided to the participant and verbally explained. This included explaining the purpose of the study, as well as their ethical rights as a research participant including that their participation was voluntary, that they could withdraw at any point without explanation, and that all information was confidential and anonymous except as required by law. Written or verbal permission from the participants was then sought. In the case where there may have been language issues, a cross (' $\mathrm{x}$ ') or a verbal permission as verified by an interpreter, was sufficient as a signature and the researcher signed as the witness. Proceeding this, permission to record the interview was sought for the purposes of accurate transcription.

After the interview was conducted, the CALD family participants were asked to complete a short demographics survey collecting data on age, sex, ethnicity, and common local services that they access, and the DHS caseworkers and case managers were asked to complete a short demographics survey collecting data on age, sex, ethnicity, length of job, and self-rated experience with different ethnic groups in the CPS. Only the CALD family participants were reimbursed with $\$ 50$ after the interviews were completed, as a sign of appreciation for their time and to cover any transport cost if applicable. After all the interviews were completed, they were then transcribed and themed.

\subsection{Sample}

2.3.1 CALD families

2.3.1.1 Ethnic background 
The 29 CALD families that took part in this study came from a diverse range of ethnic backgrounds ${ }^{5}$. These included: (i) Arabic-speaking and Middle Eastern countries (coded as 'ARB') - Egypt, Iraq, Jordan, Lebanon, and Turkey, (ii) Asian countries (coded as 'ASN') - Cambodia, Vietnam, and Philippines, (iii) African countries (coded as 'AFR') - Sudan, Burundi, Ethiopia, Ghana, and Sierra Leone, (iv) East European countries (coded as 'EEURO') - Greece, Macedonia, and Serbia, (v) Pacific Island countries (coded as 'PAC') - Maori New Zealand and Samoa, (vi) South American countries (coded as 'SAM') - Argentina and (vii) West European countries (coded as 'WEURO') - Netherlands.

\subsubsection{Sex, age, generation, and citizenship}

Ten participants were male (34.5\%). All interviewees varied in age from 22 to 67 years (Mean $=42.2$ years). Two participants were born in Australia and thus are second generation Australian. At the time of the interview, 20 participants were Australian citizens, four were permanent residents, three were refugees, and one was a temporary resident ${ }^{6}$. The number of years participants lived in Australia varied from one to 45 years $($ Mean $=$ 18.9 years).

\subsubsection{Self-identified ethnicity}

Five of the 28 participants that completed the survey question described their ethnicity as 'Australian'. Seven participants hyphenated their ethnicity to indicate dual ethnic identity (such as, 'Burundian-Australian' or 'Australian-Arabic'). The remainder identified their ethnicity in line with their culture of origin, e.g. 'Vietnamese' or 'South Lebanese'.

\subsubsection{Use of community services and programs}

CALD families sourced various services and programs in the community. These included language centres for interpreting and translating $(n=4)$, parenting programs $(n=6)$, Migrant Resource Centres $(n=5)$, Ethnic Communities Councils $(n=4)$, employment centres such as Centrelink $(n=11)$, childcare $(n=13)$, local cultural centres $(n=5)$, and local youth services $(n=7)$.

\subsubsection{DHS caseworkers and case managers}

\footnotetext{
${ }^{5}$ Note: No names of interviewees have been reported here to protect their confidentiality and anonymity.

${ }^{6}$ Survey data on this question was only collected from 28 of the 29 participants.
} 


\subsubsection{Ethnic background}

Five of the 17 participants were from a Middle East and North African background, including Afghanistan, Egypt, and Lebanon. Five participants were of South or South East Asian background, including Burma, Laos, Philippines, Vietnam, and India. One participant was from a South American background, namely Uruguay, and one was from a Sub-Saharan Africa, namely Ghana. Finally, four participants were from an Anglo Saxon background, and no Indigenous participants took part in the interviews. All caseworker and case manager participants are coded as ' $\mathrm{CW}$ '.

\subsubsection{Sex, age, and generation}

The DHS interviewees ranged in age from 23 to 59 years (Mean = 33.9 years). Only two participants were male $(12 \%)$ but this is representative of the social worker population in Australia. Eight of the participants were born in Australia, and of these, four were of Anglo Saxon background.

\section{$\underline{\text { 2.3.2.3 Self-identified ethnicity }}$}

Three of the 17 participants described their ethnicity as 'Australian' or 'Anglo Australian', and all of these were of Anglo Saxon background ${ }^{7}$. Six participants indicated a dual ethnic identity (such as, 'Asian-Australian' or 'Australian of Indian background'). One participant identified as 'trans-racial'. The remainder identified their ethnicity in line with their culture of origin, e.g. 'African' or 'Latin American'.

\subsubsection{Job role and length}

Participants identified their job title as 'caseworker' $(n=6)$, 'child protection caseworker' $(n=3)$, 'Brighter Futures $^{8}$ caseworker' $(\mathrm{n}=1)$, 'Multicultural caseworker' $(\mathrm{n}=3)$, 'caseworker specialist' $(\mathrm{n}=1)$, and 'Manager case work' $(n=3)$. The number of years working at DHS in their current role varied from one month to 14 years $($ Mean $=3.8$ years $)$.

Note: In NSW, caseworkers (child protection, early intervention, and/or multicultural caseworkers) and caseworker specialists report to case managers ('manager case work'). In Australia, a caseworker has at least a three-year undergraduate degree and a case manager is internally appointed within the CP agency based on their

\footnotetext{
${ }^{7}$ One Anglo Saxon caseworker did not complete this question on the survey.

${ }^{8}$ Brighter Futures is the name of DHS' early intervention program for at-risk families.
} 
professional experience. It is unknown the extent to which cultural competency is taught in undergraduate degrees and different universities would place emphasis in their own unique ways. However, all new staff employed by the NSW DHS receive some initial training on cultural competency.

\subsubsection{Self-rated experience with the needs of CALD, Indigenous, and Anglo Saxon families in the CPS}

Eleven participants identified themselves as having significant experience with or knowledge of the needs of families from a Middle East and North African background; five identified having significant experience or knowledge of families of North East Asian background; seven with families of South East Asian background; four with families of Pacific Islander background; and one with families of South East European background.

One caseworker identified that they did not have significant experience or knowledge of families of CALD background, and another identified that they did not have significant experience or knowledge of families of Indigenous or Anglo Saxon background. Finally, one caseworker felt they did not have significant experience or knowledge of families from all three backgrounds - CALD, Indigenous, or Anglo Saxon background. These self-rated perceptions may, in part, reflect reporting biases that more demonstrate their confidence to deliver services to families from these groups.

\subsection{Methodological limitations}

In Australia, the child protection system includes early intervention, child protection, and out of home care (OOHC). Thus, one main limitation of this study is that it is unknown which of these CALD families and caseworkers are commenting on. However, it is known by the research anecdotally that caseworkers mostly spoke of child protection itself as they often explicitly clarified if they were talking about early intervention or OOHC.

\section{Results}

Results have been presented here in two sections: (i) whether and why CALD families prefer an ethnic-match, and (ii) whether and why caseworkers prefer an ethnic-match. In this way, areas in which services users and service providers either overlap or differ can be identified and thus better inform how to overcome any gaps in current service delivery.

\subsection{Whether and why CALD families prefer an ethnic-match}


There was incredible diversity in response to whether CALD families prefer an ethnically-matched caseworker. Some preferred a match, others preferred a non-match, and others yet had no preference at all. This diversity indicates that there is large individual variation among CALD families, and that as a result, offering CALD families a choice about whether they would like a matched caseworker is crucial to good practice.

In other words, it cannot be assumed that just because a family is of CALD background that they will or will not prefer to have a caseworker of the same ethnic background as themselves. Having said this, the reasons behind why CALD families may or may not have a preference are identifiable, and it is important that caseworkers are aware of the issues that may impact their choices and preferences. This kind of cultural awareness, and offering choice, are demonstrative of cultural competency in child protection practice.

\subsubsection{Preference for an ethnically-matched caseworker}

The results indicate that there are two main reasons why CALD families prefer an ethnic match: (i) to overcome language barriers if English proficiency is an issue and (ii) to provide sensitivity to cultural and other needs. These two factors replicate themes identified in the literature review and are described more fully below.

\subsubsection{To overcome language barriers}

Repeatedly, most participants cited lack of English proficiency as the main reason for a preference of an ethnically-matched caseworker. For example, some of these CALD family participants said:

They think it's better to be Macedonian ... easier because of the language [EEUR_2]

How I can explain in English, what I real feel? Same my country, I can express what I feel, he can understand [ASN_2]

I need to explain everything of my culture, but if they are the same, there's no need because he know everything about my culture [ARB_3]

If the caseworker is the same, Vietnamese, very easy to understand... [and] explain very clearly ... I can talk to them. But Australian, so-so [ASN_3]

The [caseworker] that I am involved with at the moment speaks Arabic and from [the] same religion, so I find it very easy to converse, she can understand me a lot better [ARB_4] 
If the caseworker is from the same origin and speak the same language, it is a lot easier. I just recently got a Vietnamese caseworker, but prior to it, I got English speaking caseworker, so it has been more difficult for me to try to explain to them everything ... It's difficult to say [what] I'm thinking [ASN_4]

[My wife] don't have any English language, so she can't understand. When she have problems with the Department of Community Services, I will come between like [an] interpreter. [But] I don't have more knowledge about what you [DoCS] do, I'm just interpreting. So she will ask me, and I'll pass it to you, and then you say your word and I'll pass it to her. From here, we will keep rotating without getting each other... But if Sudanese, they can make it direct. They understand [the] ways Sudanese bring up their children and she knows the system in Australia, so she can figure out the right direction you can go in [AFR_1]

Importantly, these findings show that CALD client families who are able to speak in their native language are not just able to accurately and fully explain their feelings, but they are also saved the need to explain cultural factors. Thus, and not surprisingly, it seems that overcoming language barriers is significantly related to overcoming cultural ones.

\subsubsection{To provide cultural and general sensitivity}

Regardless of whether language is a barrier, CALD families may prefer an ethnically matched caseworker because they believe they are more aware of and sensitive to their cultural needs. Thus, the benefit of cultural awareness and sensitivity here is explicit. Quotes from all CALD family participants who made this point are below:

Caseworker to be Sudanese is the main important thing to me ... He or she will have a bit of knowledge culturally [AFR_1]

I been trying to explain, but sometimes it's a bit hard because he doesn't understand [my cultural needs]. That's why I prefer the Samoan caseworker ... Same [is] better [PAC_2]

Same [caseworker] is better, because our culture have different religion, different tradition, different food. Muslim is not to eat ham, pork, drink beer, whiskey. When DoCS take our kids for another culture ... Our kids, they eat maybe there [ARB_2] 
Interestingly, this study found that in addition to cultural sensitivity, an ethnically matched caseworker can provide an overall or general sensitivity to that family simply because of the similar ethnic background. Two CALD family participants explicitly made this point saying:

I think there is a bit of difference. The English speaking caseworkers just fulfil what they got to do. I sense that the caseworker of Vietnamese origin [has] dedicated more attention to my problems [ASN_4]

Emotionally you can feel if it is [a] different person ... [otherwise there] may be discrimination [AFR_1]

One other participant made this point implicitly saying, "All Vietnamese caseworkers, all good ladies" [ASN_6]. This participant was indicating that she believes caseworkers from her culture are better than caseworkers from other cultures because they are more understanding or sensitive.

Thus, an ethnically matched caseworker may provide both cultural and general sensitivity.

\subsubsection{Preference for a non ethnically-matched caseworker}

Although CALD families may benefit from having a caseworker who speaks the same language, who understands cultural factors, and/or is more sensitive to them, some CALD families may prefer a non-ethnic match. There were four main reasons that underpin such a preference: (i) to maintain confidentiality from caseworkers belonging to the same community as the client family, (ii) to avoid caseworkers that originate from areas in which they have regional conflicts, (iii) to prove they are not hiding any information, and (iv) to learn or practice their English. The last two of these have not been reported previously in the literature. All four factors are described more fully below.

\subsubsection{To maintain confidentiality}

Some CALD families may opt for a non-ethnically matched caseworker if they fear a breach of confidentiality. This is a fundamental issue for collectivist families interested in protecting the family name and standing in the community. Several CALD family participants made this point. As examples, some said:

We have problem with our culture - don't speak too much, it's bad! That's why, maybe better outside [ARB_1] 
Definitely not [a matched caseworker]! Not just Ethiopian, not [even] African [caseworker]. Privacy very important. I feel 100\% comfortable with my caseworker. I think [she] is from India ... It's a health issue. I may be suicidal [AFR_3]

Someone [caseworker] different. I like my people, but they don't understand [my situation]. I don't know why? [It] make me so stressed. Sorry what I say, I like my Serbian culture, my people, but this situation make me so stressed. I like more Australian [caseworker] [EERUO_4]

[I prefer] different [caseworker]. Something about communicating with the same nationality [about our family], [I] don't like to. It's not just with DoCS, it's with everything. We don't like to communicate with them [Lebanese]. So rather Aussies [caseworkers], not Lebanese [ARB_5]

Someone [caseworker] who is different. I want to maintain my private and confidentiality. I trust [other] people more than in my own culture. The side effect when you reveal things [is that] some [people] in our community would say something [AFR_5]

I'd like to have a Samoan caseworker because of the language, but the problem is I don't trust that person. I can speak and understand [English] but not all of them [words]. I need to understand and know more about the situation, but I need to have a different [caseworker] [PAC_1]

These results show that the fear of a breach of confidentiality is strong among CALD groups and can have serious consequences for individuals, varying from stress to suicidal ideation ${ }^{9}$. They also show that if this fear is greater than the benefits of matched caseworkers - to help overcome language barriers and provide (cultural and/or general) sensitivity - then CALD families may prefer a non-ethnic matched caseworker.

\subsubsection{To avoid people with whom they have regional conflicts}

Another reason why CALD families may prefer a non-ethnically matched caseworker is to avoid caseworkers who come from areas with whom they have regional conflicts. One participant said, "Northern Sudan is different. We were in a war for 21 years and that's why we are here in Australia. So if someone [caseworker] come from Northern Sudan, it'd be worse. You'll be more farther than Australian people ... Chinese or Lebanese

\footnotetext{
${ }^{9}$ Unfortunately due to word limitations, extensive contextual information for these quotes cannot be provided.
} 
or anyone [is] better!" [AFR_1]. However, of note, is that only one participant made this point therefore this reason may not necessarily be a common one.

\subsubsection{To prove they are not hiding information}

Although this issue did not emerge in the literature review, we found evidence that CALD families may prefer a non-ethnic match because they can then prove they are not hiding anything from caseworkers. However, only one participant made this point saying, "I don't prefer Lebanese [caseworker] cos I'm not hiding anything. Whatever she is or whatever she speak, I don't care, because they're doing this job and everyone the same ... She [caseworker] came with Lebanese girl and ask me if I prefer her. I said, 'no, I seen you before, why change? I'm happy with you' ... She said, 'maybe you be more comfortable?' I said 'no, what's here is open to everyone, I'm not hiding [anything]"' [ARB_9]. Thus, this reason may not be a common one.

Importantly, however, this quote shows that while caseworkers are motivated to provide a culturally sensitive service, CALD families may reject this option to enhance their appearance of transparency and engagement with the child protection system. While only speculative, it is possible that CALD families do this if they believe that engaging with a non-ethnically matched caseworker will make it increase the chances of their child/ren being returned to them quicker.

\subsubsection{Opportunity to learn English}

Another, less common reason why CALD families may prefer a non-ethnic match, and which was not identified in the literature review, is so that they have the opportunity to learn and practice English. Only one participant said, "[I prefer] different. I wish I have all friends from all cultures. When I learn something, it's good ... Maybe I will learn English. With the relatives or friends, same talking, same same same. Routine, it's no good" [ARB_3].

Importantly, this result shows that although not a common issue, for those CALD families for whom this is relevant, they may be unaware of the magnitude of any child protection intervention and that if this is the case, caseworkers should stress the importance or necessity of using an interpreter or bilingual staff member. Indeed, child protection matters are complex and far more important than providing an opportunity to speak English, and CALD families (albeit a few) may not realise this. 


\subsubsection{No preference regarding ethnic-matching}

There were a number of participants who indicated that they did not mind whether they had an ethnicallymatched caseworker or not. This occurred when there was: (i) a general indifference, (ii) language was not a barrier, (iii) a belief that all caseworkers are equally trained/skilled, and (iv) a belief that good/kind caseworkers can be from any cultural background. Examples of these are provided below.

\subsubsection{General indifference}

Individual differences can cause some people from CALD groups to have a general indifference about whether their caseworker is from the same ethnic background as themselves. Two participants made this point saying:

I really don't mind [SAM_l]

I don't mind either way, the same culture or different. I have no problems with either culture [PAC_1]

Importantly, this result highlights that the needs of CALD families are not just cultural or linguistic in nature and that individual differences need to be considered as well. Here, individual differences refer to the preferences of individuals that cannot be attributed to other factors.

\subsubsection{Language not a barrier}

A significant factor that influences whether CALD families prefer an ethnic match is language; if English proficiency is not a barrier, then CALD families are not likely to need or prefer an ethnically matched caseworker. Quotes indicating indifference to ethnic matching because language was not a barrier were made explicitly by three participants:

I don't mind ... Only if you can 't communicate to understand, then that's all you need [AFR_4]

I don't mind, cos I speak English. If I didn't, I would say I want [a matched caseworker] cos it's easier ... to explain things [ARB_7]

It doesn't matter that the caseworker is Australian-born or whether he comes from a different culture, as long as that person uses an interpreter in dealing with him [AFR_2] 


\subsubsection{Belief that all caseworkers are equally trained/skilled}

Interestingly, this study found that CALD families may be indifferent to the issue of whether they would like an ethnically matched caseworker if they believe that all caseworkers are equally trained/skilled in both child protection practice generally, as well as in delivering a culturally appropriate service delivery; thus, the ethnicity of the caseworker is seen as irrelevant to child protection practice. This finding has not been reported in previous research but is perhaps not a common issue as only two participants made this point. These two example quotes are provided below:

Doesn't matter [if they are the same or not]. Most important, if she's experienced enough to deal with different background, different cultures [ARB_8]

[Caseworker]'s doing a good job, so [I] wouldn't change anything. They do what I ask. Everyone is the same in their job, they know what they're doing. Doesn't matter if you're white, black or green ... they must get taught at school or whatever, so they [caseworkers] all the same [WEUR_1]

\subsubsection{Belief that good/kind caseworkers can be from any cultural background}

This study also found that CALD families may be indifferent to whether they would like a matched caseworker if they believe that good/kind caseworkers can be from any cultural background; again rendering the ethnicity of the caseworker irrelevant. This finding has also not been reported in previous literature and again, may not be a common theme. The two participants who made this point said:

Anyone, because not all persons the same. Sometimes I have people not my culture, who are very kind, very good. Sometimes I have my culture, it's bad! [ARB_1]

They should know what your needs are, they should listen to you. Maybe someone, you know, not even from your [own] background might listen to you, so all the same. It's a mixed country [ARB_7]

\subsection{Whether and why caseworkers prefer an ethnic-match}

Two caseworkers identified specific CALD groups as having a preference for an ethnically matched caseworker. They said: 
[The] majority will say 'I want someone that is Vietnamese'. That's my experience [CW_8]

Some families will prefer the same. [For example] Greek family will prefer to have a Greek caseworker [CW_12]

Thus, there may be some CALD groups that have a tendency to prefer an ethnic match. Nevertheless, there was diversity in response to whether caseworkers preferred an ethnic match, a non-ethnic match, or had no preference at all, similar to the CALD family participants. Responses from these three categories are described more fully below.

\subsubsection{Preference for an ethnically-matched caseworker}

Some caseworkers expressed a definite preference for matching CALD families and caseworkers. As examples, some caseworkers said:

It is good if we're from the same background and understand each other. We can help them better [CW_1]

Definitely better to match, otherwise you get the closest mix. [For example] if you had a Cambodian family but you don't have a Cambodian caseworker, you go for Vietnamese [or] Thai [CW_2]

I think the best thing is to have a [caseworker] from the same background. If not, at the very least consult with a person of that same cultural background, and say, 'what do you think about this?' or 'how do you think I should approach that?'Yeah, it's not the same as having the same caseworker" [CW_11]

Responses from caseworker interviewees indicate that the two main reasons why caseworkers may prefer an ethnic match are for language and (cultural) sensitivity issues. This corroborates the findings that emerged with the CALD family participants.

\subsubsection{To overcome language barriers}

Like the CALD family participants, most caseworkers primarily preferred an ethnic match for language reasons. As examples, caseworker interviewees said:

In terms of language interpretation, it's great [CW_14] 
A lot of the time it will be based on just language issues [CW_8]

However, one caseworker did also note that there can be some work practice issues when a matched caseworker is used for language reasons, saying "It can be complicated when you go out with someone and they don't translate back into English so you can’t write the notes. It happens sometimes. Depends on who it is" [CW_14].

Thus, matching caseworkers for language reasons is useful for meeting the needs of CALD families. However, there is a need to ensure appropriate follow up in the workplace, so that all caseworkers and case managers involved in that case are fully informed.

\subsubsection{To provide cultural awareness and sensitivity and general sensitivity}

Consistent with the CALD family participants, most of the caseworkers also acknowledged that providing a matched caseworker is helpful for being aware of cultural factors and meeting the cultural sensitivities of their CALD clients. For example, some of these caseworkers said:

The pros are the knowledge base, the sensitivity that they know how to approach that family [CW_15]

If a family has a caseworker that is from the same culture, they are going to have more understanding in terms of language and mannerisms, and how they go about everyday life [CW_7]

At least that caseworker knows, they've grown up in this culture, they know the views of this culture, what their beliefs are, and it would help. Maybe the family would respond better having someone there, 'oh, you understand me' [CW_9]

I might think feet-binding is very bad. I might discredit her [mother], [but] in fact she was doing with good intention to provide better care for that child. Misunderstanding of the culture would be the disadvantage $\left[C W_{-} 1\right]$

In any culture that follows Islam there's certain rules they have to follow. It depends on how strict the family is with the religion. Generally, a woman isn't allowed to even shake hands or speak to a male that isn't from her family or isn't married to her so that's very important I think [to know] [CW_7] 
Thus, providing a culturally aware and sensitive service can help enhance engagement with the child protection process and accurate understanding of a child's wellbeing. Interestingly, however, two caseworkers also acknowledged that simply providing a caseworker from any CALD background was helpful for providing a general sensitivity to CALD clients. They said:

I found that even though I'm not from the same cultural group as various clients or communities in the area, the very fact that I can speak a similar language or understand some of the norms, makes it a lot easier for them [CW_8]

We're [CALD caseworkers] more approachable compared to other races. I think a lot of CALD caseworkers are more adaptable or more understanding of other cultures. They are better at working with other cultures. Whereas Anglo caseworkers only know one language. They don't understand that much [about how] to work with CALD communities. They are not that adaptable. I can give you [an] example. I used to go out with [an] Anglo-background caseworker to a CALD family. She has three kids under five. She doesn't work, but her husband works full time. She's basically on her own with the kids. Her house is a little messy with toys on the floor, clothes, but the children have food in the fridge and the children are well cared for. To me, that is good. Ok, three kids [are] not easy. I'm a mother. This caseworker recommended that this is neglect, and mum doesn't have parenting skills ... We see things different, probably because of our background and experiences. We are more accepting, flexible on making judgement on people. [The] consequences of those judgements are very, very harmful. Sometimes it's damaging to the family ... Also the basic standards that we expect from our clients are different. Probably, we come from poorer countries, as long as the children are happy where they are ... Whereas people who have a higher standard, expect our clients to have a higher standard for their children $\left[C W_{-} 1\right]$

Thus, these results show that matching caseworkers and CALD families may provide cultural awareness and sensitivity as well as an overall sensitivity to the experience of CALD groups; a general cross-cultural adaptability of CALD caseworkers perhaps born of their own daily lived cross-cultural experiences. Moreover, these benefits can enhance the accuracy of risk of harm assessments to children from these groups because they help avoid the use of one standardised ideal of parenting, often reflecting the dominant (socio-economic) culture, from which to judge all families. 
In other words, a lack of cultural awareness and sensitivity can be detrimental to the well-being of a CALD child and at worst, reflect discrimination. Indeed, one caseworker said, "As they [non-CALD caseworkers] become a more experienced worker working with CALD communities, I think they become better at it. If they're new caseworkers and don't know much about other cultures, and also if they come from a higher socioeconomic, then they can become very judgemental and make bad decisions" [CW_1]. Thus, matching caseworkers may help avoid such serious consequences.

\subsubsection{Preference for a non-ethnically matched caseworker}

Four reasons for why caseworkers prefer a non-ethnic match were cited. These were: (i) to help avoid a fear of a breach of confidentiality, (ii) to prevent over-identification between CALD families and caseworkers, (iii) to protect CALD caseworkers from community backlash, and (iv) to learn about other cultures. These are described more fully below.

\subsubsection{To avoid a fear of a breach of confidentiality}

The main reason why CALD families preferred a non-ethnic match was to avoid a fear of breach of confidentiality. This was corroborated by several caseworkers, and as such is demonstrative of cultural awareness and sensitivity. Examples of this include:

The cons are, and it's fairly obvious, the community backlash; the possibility of knowing that family [CW_15]

With small communities, they prefer not to have someone [caseworker] from their community, from their culture [CW_12]

In other cultures or other families, some don't want somebody from the same nationality or from the same background as them, because they fear this is going to spread around the community [CW_4]

Every case needs to be addressed individually. Sometimes it does help when they can speak the same language, and at other times they are from a similar community and it's not appropriate [CW_17]

Sometimes they prefer to have someone who speaks English because of their family face. Because it's a very small community, you can meet them at every social event, they have a very significant face in that community, they feel they have loss of face [CW_5] 
Some families will go, 'no, no, no, I don't want anyone from my culture', because they don't want people finding out about what's going on. There's all sorts of confidentiality issues in a lot of ethnic groups [CW_8]

Thus, these results confirm previous literature that the fear of a breach of confidentiality is a significant issue for CALD families and that as such, non-matching is an important part of good practice with this client group.

\subsubsection{To prevent over-identification}

Although a fear of a breach of confidentiality is the main reason why CALD family participants prefer a nonethnic match (and that caseworkers are aware of this), the main reason why caseworkers prefer a non-ethnic match is to avoid possible over-identification between CALD families and caseworkers. Indeed, no CALD family participants cited this issue, yet several caseworkers noted this as a significant barrier to effective child protection practice. Examples include:

Over identifying with families is good in a way, to build a rapport, but later it becomes a dependency, where I think the boundaries are very blurred [CW_2]

There is a caseworker here who is Pacific Islander, who will very much downplay the issues of a family, because she will see it as culturally acceptable and we see it as risk. I think there is absolutely the potential for that. It comes down to the skill of the worker and the skill of the manager ultimately [CW_15]

You have to go case by case because some relationships you have to form, that might be the only common ground they have to work with the client, and that can be a very useful way to engage the family. But in other situations, the lack of identification with some of our staff would avoid bias and opinion [CW_14]

I think [a] different [caseworker] because you need to have someone who is objective. I think if you put a Samoan or Tongan caseworker with a Samoan or Tongan family, and the issue is physical discipline, you might get a lot of minimalisation of the issues ... Definitely to consult and go out with, but not on an ongoing basis. I think the lines between $C P$ and culturally appropriate behaviour could be blurry [CW_16]

Based on my experience, I think there are advantages and disadvantages with race-matching caseworkers and clients ... The disadvantage is that sometimes caseworkers over-empathised with their clients or we might overlook the child protection issues. We'll put our cultural needs above the child protection needs. Some 
caseworkers want to minimise the issues because they're working with their own community ... [and have a] very good relationship with the family after working [for] a while [with them] [CW_1]

Thus, over-identification is seen as a possibly significant risk to the accurate assessment of a child's well-being. The overall/general sensitivity that CALD caseworkers can provide CALD clients, which in turn can enhance accurate risk of harm assessments and avoid possible discrimination, can also be problematic in that risk of harm may be downplayed or minimised. This indicates that there is an irreconcilable difference in regards to ethnic matching; it can both enhance and compromise accuracy in risk of harm assessments. As a result, and consistent with the suggestion of several caseworkers, this study proposes that the advantages and disadvantages of ethnic matching be assessed on a case by case basis.

Having said that, over-identification can occur not just among CALD families but also among white Anglo families, and indeed between any service user and service provider that share a common experience. Two caseworkers made this point, saying:

I think that happens quite a bit [over-identification] across all cultures, even in Anglo families. Especially cos Anglo families can express themselves a lot clearer and their beliefs and values are clearly known, we tend to be able to relate to them quite well as a Department, [so] we do think about their [parent's] loss, and it can cloud your judgment if you're not really really careful [CW_8]

I think it [over-identification] can happen a lot. Not just with CALD clients, just with clients in general in this sort of work. I guess it's hard for caseworkers to distance themselves if they do feel a connection or they have experienced the same thing or been down that same road [CW_9]

These quotes are crucial for highlighting that over-identification is not just a risk for CALD families and that arguably, if caseworkers assume this is the case, that they are minimising the importance of delivering a culturally sensitive service. In other words, caseworkers and case managers who see over-identification as a serious risk and thus have an overall preference for non-ethnic matching, may be underestimating the value and necessity of the cultural sensitivity that ethnic matching provides. Importantly, we did not interview any caseworkers who held such a view. Indeed, all caseworkers who cited over-identification as a risk to good practice also had a preference for assessing the merits of ethnic matching on a case by case basis. Nevertheless, 
emphasising the risk of over-identification is, in principle, a threat to the delivery of a culturally competent service.

Several caseworkers noted that good mentoring from case managers can help overcome the risks associated with over-identification. As one interviewee said, "It's part of the job as a manager - you need to be on top of [it]. You need to be aware, through supervision. If you are noticing that [over-identification] then you need to be discussing that with your caseworkers" [CW_9].

Thus, over-identification appears to be the main reason why caseworkers prefer not to match with CALD families, and managing the risks associated with it is seen as part of good social work practice. Notwithstanding, it is important that case managers do not downplay or overlook the importance of cultural sensitivity as part of this 'risk management'. Moreover, it is not just the job of management; it is the role of CALD caseworker themselves to also ensure they manage this risk. As one caseworker said, "As long as you keep in mind what your role is - Why are you doing this job? Why are you assessing this family? - You won't be having any problems" [CW_1]. Importantly, the issue of 'over-identification' with respect to race has only been reported once in previous literature (Gray, 2002) and yet was a common and significant finding in this study.

\subsubsection{To protect CALD caseworkers from community backlash}

As identified in the literature review, CALD caseworkers may prefer a non-ethnic match to avoid issues that would otherwise arise from an ethnic match. Three caseworkers made this point:

A lot of caseworkers know the families we are dealing with and it might be a conflict of interest [CW_16]

I think a lot of people within the community know each other ... If they are from that community how are they [caseworkers] going to be perceived? Do they know them? Are they related to this family? I guess in that sense, it might not be a good idea just on the off chance you do know this family. You do need to be aware of that [CW_9]

I think that in an ideal world having that matching would be really useful for the clients, but from a case work perspective ... If my client is Egyptian and they know I'm Egyptian there's all sorts of issues about me busting in there and taking their kids. Like that's going to end up at my dad's house, you know what I mean? And that would be the same for various other caseworkers [CW_8] 
Thus, another benefit of non-ethnic matching is that it can protect CALD caseworkers from possible backlash in their community.

\subsubsection{To learn about other cultures}

A final, and less commonly cited, reason why caseworkers may prefer a non-ethnic match is that it becomes a direct way of learning about different cultures. In turn, it can contribute to the development of their cultural competency but it can also ease a resource burden if there are not enough CALD caseworkers. Only one CALD caseworker made this point, saying, "I would prefer that other caseworkers have the opportunity to work with CALD families more. Otherwise we would be just stuck. What if we don't have enough people who are from those particular cultures? Then those people, those caseworkers, are not going to have the experience or knowledge. And I think, not only do the families have to come out of their comfort zone a little bit [but also the caseworkers] ... It's breaking that barrier" [CW_7].

\subsubsection{No preference regarding ethnic-matching}

Several CALD families expressed that they did not have a preference regarding ethnic matching. In comparison, very few caseworkers said that they were indifferent to whether CALD families should or should not be matched. In other words, caseworkers generally understand that there are pros and cons to ethnically matching CALD families and caseworkers, and tend to reconcile these on a case by case basis; and even then, caseworkers may express a slight preference for either matching or non-matching.

For example, one caseworker said, "I guess there are positives and negatives on each end ... [but] I'd probably say to [ethnically] match if ideally we had the resources to do that ... I do think families respond ... a lot better to a caseworker that is from their culture. It's understandable. They feel like this person is going to understand them and are going to be able to relate to them. I guess the caseworker just needs to be clear on what their role is, so that the family doesn't identify them as a friend or a support when that caseworker is actually there as an authoritarian figure" [CW_9].

On the other hand, another caseworker said, “I think there's pros and cons really ... [but] I personally think that probably not [ethnic-matching]. Having someone there as a point of contact to bounce ideas off and reflect on is probably a bit better than putting a family in a situation where it may not work ... In [CSC], it caused some problem in terms of, often caseworkers living and working at the same CSC and knowing the family. There are 
some really large cultural groups, like Vietnamese or Chinese, where it may not be an issue. [But] an Afghani cultural group, I don't think it would work" [CW_15].

Overall, however, the importance of looking at the individual family was emphasised by caseworkers. In the words of one interviewee, "I don't think we should generalise ... Every family is different, everybody’s personality is different" [CW_4]. This caseworker also went on to say, "You say 'look, we are going to be working with you, what would be best? Do you think you would benefit more from someone from your cultural background to be working with you or are you happy with myself?' Giving them that option if we have that resource" [CW_4]. Thus, as a result of individual differences, it would be ideal if caseworkers could offer an option as to whether they prefer an ethnically matched caseworker (pending resource constraints).

\section{Discussion}

There were three main aims of this study: (i) to explore whether themes identified in the literature review play out in an Australian sample, (ii) to compare and contrast the perceptions and experiences of CALD families and caseworkers, and (iii) to identify principles of good practice in regards to ethnic matching. The results of these aims are incorporated into the discussion below.

\subsection{Summary of results}

There were several important findings in this study. Firstly, this study found that CALD families prefer an ethnic match primarily to overcome language barriers if English proficiency is an issue. Caseworkers corroborated the importance of this factor. However, if matched caseworkers are used for language reasons, it is important that all information is translated back to other caseworkers and case managers involved in the case.

This study also found that CALD families and caseworkers prefer an ethnic match because of the cultural awareness and sensitivity this provides. In turn, these can aid in understanding cultural norms, facilitate engagement with the child protection process, enhance the accurate assessment of a child's well-being and avoid discriminatory judgements.

These results are positive; the consistency between service users and service providers indicates that there is no service gap in need of filling with regards to overcoming cultural and linguistic differences as ethnic matching does help overcome barriers that result from such differences. However, this study found that ethnic matching 
carries two significant risks to effective child protection practice and it is here that gaps in service delivery may occur.

One risk to effective child protection practice is a resource issue; there may be an insufficient pool of caseworkers trained in cultural competency to draw on if CALD families request a non-matched caseworker. CALD families may request a non-matched caseworker because they fear a matched caseworker will 'leak' their private family matters to their community and this can result in significant stress for CALD families. However, the mere individual variation among the CALD family participants as to whether or not they prefer an ethnically-matched caseworker indicates that offering choice is crucial to good practice with CALD groups.

Thus, being able to provide a non-matched and culturally competent caseworker is important. (Importantly, this study found that caseworkers were aware of this fear, and as such is indicative of cultural competency).

Moreover, non-matching protects caseworkers who would otherwise experience backlash in their community. Indeed, population demographics alone dictate that there will be more non-CALD caseworkers in social protection work. Thus, the capacity of the child protection system to offer choice to CALD families without compromising the quality of service they receive by a caseworker is important and would only be hindered by resource issues that constrain the provision of training in cultural competency to all caseworkers.

Interestingly, this study found that caseworkers from any CALD background can offer CALD client families a general sensitivity. This may because of a shared experience as an ethnic minority in Australia. The benefit of offering a CALD caseworker of a different ethnic background to the client family is that they can provide general sensitivity but bypass the issue of a fear of a breach in confidentiality. Thus offering any CALD caseworker may arguably also be part of good practice with this group. However, this is still a 'diluted' form of ethnic matching and so does not wholly overcome issues in the same way that cultural competency training can.

Although the sample size of caseworkers is not large, the results suggest that a second significant risk to effective child protection practice is if matched caseworkers over-identify with their CALD client family and as such downplay or minimise the level of risk to a child's well-being. Thus, there can be a conflict between meeting the needs of CALD families for a culturally sensitive service and protecting CALD children from harm through accurate risk of harm assessments. CALD caseworkers themselves need to be vigilant and responsible for managing this issue. However, given that over-identification can occur in any cultural group and for any issue it is seen primarily as the responsibility of the case manager to manage this issue if it presents in case 
work. Importantly, case managers should not downplay or overlook the importance of cultural sensitivity as part of this 'risk management' as this can also compromise the child's welfare.

Given that ethnic matching has the potential to both enhance and compromise accuracy in risk of harm assessments, this study proposes that the advantages and disadvantages of ethnic matching be assessed on a case by case basis. Indeed this principle of good practice is supported by the fact that the needs of CALD families should not be generalised, and individual differences matter in the provision of a service tailored to a particular family's needs. In other words, diversity both within and among cultural groups does not make a 'one-size-fitsall' approach useful; generating heuristics such as 'ethnic matching families and caseworkers is better than nonethnic matching' are not appropriate for meeting the needs of CALD families.

This study also found that other, less common reasons why CALD families may prefer a non-match include: to avoid caseworkers that originate from areas in which they have regional conflicts, to prove they are not hiding any information, and to learn or practice their English. With regards to the last of these, it is important to highlight to CALD families that any child protection intervention is complex and important and thus they should offer an interpreter or bilingual caseworker anyway. Importantly, these results show that there a range of reasons that underpin a CALD family's preference for a matched or non-matched caseworker. Also, the last two of these three reasons have not been reported in previous literature and are new findings of this study.

Reasons why CALD families may be indifferent to the ethnicity of the caseworker include a general indifference, language is not a barrier, a belief that all caseworkers are equally trained/skilled in child protection work and the delivery of a culturally appropriate service, and a belief that good/kind caseworkers can be from any cultural background. These represent new findings as they neither replicate nor contradict previous research.

Besides avoiding the fear of a breach of confidentiality and to help prevent over-identification, caseworkers may also prefer non-ethnic matching so that the pool of caseworkers competent at addressing cultural issues is large and not wholly dependent on CALD caseworkers. Direct exposure to CALD families is a form of cultural competency training for non-CALD caseworkers, and working with families of several different backgrounds is useful for overcoming resource shortages when matched caseworkers are not available to offer to CALD families. Indeed because there will be a significant proportion of CALD families who will prefer a non-ethnic match, the need for non-CALD caseworkers to be able to deliver a culturally aware, sensitive and competent 
service is crucial. However, that non-ethnic matching offers caseworkers the opportunity to learn about other cultures was not cited as often as the other benefits of non-matching in this study.

\subsection{Putting it all together: Triangulating the data to make practice and policy recommendations}

By triangulating this data, it seems that caseworkers who perceive over-identification to be a significant risk will prefer to have a non-matched caseworker assigned to a CALD family. These caseworkers may believe that cultural sensitivity and child safety are opposing goals, and that meeting one may be at the cost of meeting the other. On the other hand, caseworkers who believe that by being culturally sensitive they will be better able to protect the child's welfare (and thus that these two issues are embedded within each other, rather than being opposed to each other), may see that offering choice to the CALD family about whether they prefer an ethnic match or not is more important than managing the risk associated with over-identification.

This study proposes that the needs of CALD families should be a foremost priority because in meeting this cultural need, the effectiveness of future and on-going intervention will be significantly and positively influenced by the extent to which child protection workers demonstrate cultural awareness and sensitivity in the initial meetings. Thus, it is proposed that firstly, CALD families should be offered choice as to whether they would prefer a matched or non-matched caseworker, (and the caseworker should explicitly explain that this offer is being made because they are aware that language, confidentiality, and cultural sensitivity may be important and relevant issues for them). If the CALD family prefers a matched caseworker, then the CALD caseworker should self-reflect and discuss with their manager on how they will address the potential of overidentification. If the CALD family prefers a non-matched caseworker, then this caseworker (like all caseworkers) should be trained in cultural competency. Finally, if the CALD family has no preference in regards to ethnic matching, then arguably it may be useful to allocate a non-CALD caseworker as this is an opportunity to provide on-site training in cultural competency. (Besides meeting the preferences of CALD families, these general principles aim to avoid confusion in case accountability that would otherwise occur with the use of two caseworkers for each case (a CALD and non-CALD caseworker, which could be another alternative guiding principle) and for the cost-efficient use of resources).

By starting with the CALD family first - and offering them an option - CALD families may feel empowered in an otherwise disempowering intervention. This form of co-operation can have positive carry-over effects that help ensure that the remainder of any intervention is as effective as possible. It also signals to the CALD family 
that caseworkers are aware of, and sensitive to, their cultural needs. As one caseworker said, "I would say most definitely the family needs to be given the opportunity to be matched up with someone from their cultural background. If they say they would prefer not, then it's not. If they say yes, which they generally will, then we need to source that for them" [CW_8].

\subsection{Future research}

One main benefit of this study is that it has addressed a research and knowledge gap in the needs and experiences of CALD families with regards to ethnic matching. However, one limitation of this study is that it has not explored the needs and preferences of white families. Indeed, one caseworker said in passing to the researcher "CALD caseworkers need to be taught about Anglo families because there are things that Anglo families say that they miss, or they misinterpret, like "I'm going to kill the little shit". They will freak out, but it's just an Aussie saying. They will want remove for that". Thus, further research into the cultural needs of white families is warranted.

Another limitation of this study is that it has not explored the needs and preferences of CALD families with biracial children. Whether and whom they prefer an ethnic match with is an area required for future research.

Finally, and interestingly, no caseworker interviewee made the point that non-matched caseworkers may underreport risk because they fear being accused of racism, or because of the risk associated with 'cultural relativism' in which abusive and neglectful behaviours are not recognised because they are also culturally normative and acceptable (e.g. Korbin, 2002; Koramoa, Lynch, \& Kinnair, 2002). Thus non-matching can also lead to riskminimisation and exploring the frequency and processes that contribute to it is also warranted for future research.

\subsection{Conclusion}

No known empirical studies have been conducted on best principles regarding whether CALD families and caseworkers should be ethnically matched in Australia. This study has begun to address this gap in the knowledge base by conducting an exploratory qualitative study into the perceptions and experiences of CALD families and caseworkers and used these as the basis for designing principles of good practice. In turn, culturally appropriate service delivery can be enhanced for this group. Moreover, the literature review revealed that overidentification in the context of ethnic matching has not received significant attention and yet was perhaps the 
most significant finding of this study indicating it is a significant work practice issue in this area.. Thus, this paper makes an important contribution to the currently scant literature. Finally, the results of this study support the trend in the literature that argues that training in cultural competency is more effective than race matching. However, it is additionally argued that part of this training in cultural competency is to ensure that all caseworkers are aware of the pros and cons of ethnic matching and thus appreciate the complexity of this issue. 


\section{References}

Chand, A., \& Thoburn, J. (2005). Research review: Child and family support services with minority ethnic families - what can we learn from research? Child and Family Social Work, 10, 169-178.

Chand, A. (2005). Do you speak English? Language barriers in child protection social work with minority ethnic families. British Journal of Social Work, 35, 807-821.

Chand, A. (2000). The over-representation of Black children in the child protection system: possible causes, consequences and solutions. Child and Family Social Work 5 (1), 67-77.

Chuan, C., \& Flynn, C. (2006). Children and young people of culturally and linguistically diverse (CALD) backgrounds in out-of-home care in NSW: Support strategies, challenges and issues. A qualitative research report. Prepared for Association of Children's Welfare Agencies (ACWA).

Courtney, M. E., Barth, R. P., Berrick, J. D., Brooks, D., \& Parks, L. (1996). Race and child welfare services: Past research and future directions. Child Welfare, 75 (2), 99-137.

Gray, B. (2002). Emotional labour and befriending in family support and child protection in Tower Hamlets. Child and Family Social Work, 7 (1), 13-22.

Harris, M.S., \& Hackett, W. (2008). Decision points in child welfare: An action research model to address disproportionality. Children and Youth Services Review, 30, 199-215.

Korbin, J. E. (2002). Culture and child maltreatment: cultural competence and beyond. Child Abuse and Neglect, 26, 637-644.

Koramoa, J., Lynch, M. A., \& Kinnair, D. (2002). A continuum of child-rearing: responding to traditional practices. Child Abuse Review, 11, 415-421.

Maitra, B. (2003). Would cultural matching ensure culturally competent assessments? In Reder, P., Duncan, S., and Lucey, C. Studies in the assessment of parenting. Brunner-Routledge, New York.

Osterling, K. L., D’andrade, A., \& Austin, M. J. (2008). Understanding and Addressing Racial/Ethnic Disproportionality in the Front End of the Child Welfare System. Journal of Evidence-Based Social Work, 5 (12), 9-30 
Owusu-Bempah, K. (1999). Race, culture and the child. In Tunstill, J. Children and the state: Whose problem? Cassell, London.

Perry, R., \& Limb, G. E. (2004). Ethnic/racial matching of clients and social workers in public child welfare. Children and Youth Services Review, 26 (10), 965-979.

Ryan, J. P., Garnier, P., Zyphur, P., \& Zhai, F,. (2006). Investigating the effects of caseworker characteristics in child welfare. Children and Youth Services Review, 28 (9), 993-1006.

Sale, A.U. (2006). Paralysed around culture. Community Care, 1614, 28.

Weinfeld, M. (1999). The challenge of ethnic match: Minority origin professionals in health and social services. In Troper, H. M., Weinfeld. M. Ethnicity, politics and public policy: Case studies in Canadian diversity. University of Toronto Press: Toronto. 